\title{
Modeling and Device Simulation of Photonic Crystal Surface Emitting Lasers Based on Modal Index Analysis
}

\author{
Guangrui Li ${ }^{\circledR}$, Jayanta Sarma, Richard J. E. Taylor ${ }^{\circledR}$, David T. D. Childs, Member, IEEE, and Richard A. Hogg ${ }^{(1)}$
}

\begin{abstract}
We present a novel semi-analytical method utilising modal index analysis, for modelling the field resonances of photonic crystal surface emitting lasers (PCSELs). This method shows very good agreement with other modelling techniques in terms of mode calculations, with the added advantages of computational simplicity, the calculation of threshold gain, and rapid analysis of finite structures. We are able to model the effect of external lateral feedback and simulations indicate that the near-field peak can be electronically displaced and the threshold as well as the frequency can be controlled through external in-plane feedback, paving the way to dynamic control of PCSELs.
\end{abstract}

Index Terms-Computational modelling, Surface emitting lasers, Photonic crystals.

\section{INTRODUCTION}

W ITH their robust, compact, light-weight and high efficiency advantages, semiconductor lasers have been extensively applied in nearly all aspects of modern society and their potential for application can hardly be overestimated [1], [2]. Various laser device geometries and structures have been designed and developed over the years with the purpose of improving almost all characteristics of the laser including high single mode power combined with narrow beam divergence and monochromatic output. The photonic crystal surface emitting laser (PCSEL) has gained particular prominence recently in achieving these characteristics [3].

A PCSEL consists of a large area two-dimensional (2-D) periodic structure which gives the PCSEL the ability to achieve large area single transverse mode operation while ensuring high power output in a uniformly narrow far-field beam [4]. As shown in Fig. 1, a 2-D photonic crystal (PC) is realised above the active region through semiconductor etch and regrowth technique to provide 2-D in-plane feedback [5], vertical emission is the result of the second order grating (2-D PC) [6]. The advantage of the

Manuscript received January 31, 2019; revised October 26, 2019; accepted October 26, 2019. Date of publication October 31, 2019; date of current version December 2, 2019. This work was supported in part by the Engineering and Physical Sciences Research Council (EPSRC) under Grant EP/K023195/2. (Corresponding author: Guangrui Li.)

The authors are with the Department of Electronic and Electrical Engineering, The University of Glasgow, G12 8LT Glasgow, U.K. (e-mail: guangrui.li@ glasgow.ac.uk; jsarmabath@gmail.com; richard.taylor@glasgow.ac.uk; david. child@glasgow.ac.uk; richard.hogg@glasgow.ac.uk).

Color versions of one or more of the figures in this article are available online at http://ieeexplore.ieee.org.

Digital Object Identifier 10.1109/JSTQE.2019.2950795

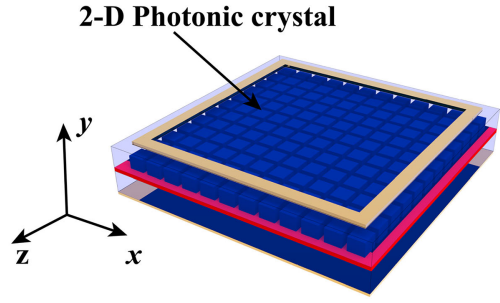

Photonic Crysal Surface Emitting Laser (PCSEL)

Fig. 1. Schematic of all-semiconductor PCSEL device. 2-D PC is fabricated above the active region by semiconductor regrowth technique. The device is electrically pumped.

PCSEL is that the drawback of using a large active area which often leads to spatial multi-moding and hence multi-wavelength lasing (as in the VCSEL [7]) is counteracted by the 2-D photonic crystal. Recently, PCSELs with high single mode power, low divergence [8], wavelength and polarisation control [9], have been experimentally demonstrated. We have also showed that coherent 2D PCSEL arrays may be readily realised [10], [11], and that external in-plane feedback significantly modifies the operating characteristics of a PCSEL [12].

Whilst experimental works has flourished, the theoretical development of PCSELs has been a daunting task. To model the PCSEL, the primary requirement is to evaluate the optical field resonances of the 2-D PC and identify the lasing mode. This is achieved through studying the wave propagation in periodic media.

The study of wave propagation in periodic media has been of considerable interest for more than a century [13]-[15]. The early use of periodic structures at optical wavelengths lead to the development of 1-D gratings and their application in semiconductor lasers such as distributed Bragg reflector (DBR) [16], distributed feedback (DFB) [17] and vertical cavity surface emitting lasers (VCSELs) [7] which have become ubiquitous. Recently, optical waves in structures with 2-D or 3-D periodicities (referring to PCs) have received intense attention. As can be expected, the analyse of a PC is considerably more difficult than evaluating the characteristics of 1-D gratings. Over the years, different methods have been developed to analysis PCs, predominantly plane wave expansion (PWE) [18], coupled mode theory (CMT) [19] and 


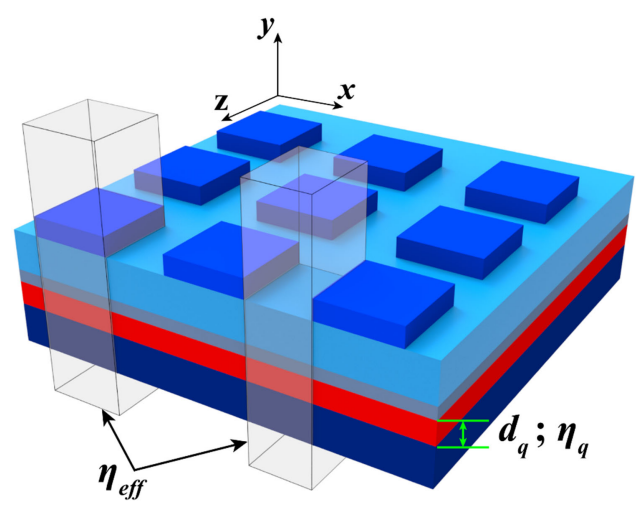

Fig. 2. 3-D Schematic of PCSEL structure.

pure numerical method such as finite difference time domain (FDTD) [20].

PWE originates from the solving technique of Hill's equation and is based on describing the field as the superposition of a complete set of plane waves with the media periodicity imposed via the Floquet-Bloch representation [21]. However, such a technique is not suitable for PCs with finite extent since the Floquet theorem is strictly applied only for infinitely extending periodic structures. Such limitation can be avoided using CMT for laser threshold analysis but the mathematical complexities are quite daunting and this method may take considerable effort to implement. FDTD is a powerful numerical tool and can handle very general conditions but does not provide any 'physical-feel' and hence is not suitable for the design of the devices. Additionally, FDTD requires significant computational resources.

Previously, we have developed an essentially analytical technique termed modal index analysis (MIA) which is relatively simple, easy to implement and requires modest computational resources [22]. In this work, we will first briefly review the MIA technique with numerical comparison as the validation of our model, then our new technique is used to describe experimental phenomenon we observed previously [12]. We go on to predict the characteristics of PCSELs of different sizes and with different strengths of external reflection. Finally, a PCSEL device with small size is proposed for better threshold margin and therefore high single-mode power densities.

\section{DESCRIPTION OF MODEL}

\section{A. Modelling Technique: $y$-Direction}

Our detailed model is published in [22] but described briefly here. In this paper, a rectangular co-ordinate system $(x, y, z)$ consistent with the rectangular device geometry is used throughout. The vertical structure is a multilayer slab achieved using semiconductor epitaxy. Here we consider a multilayer slab with thickness $d_{q}$ and given refractive indices $\eta=\eta(y)=\eta_{q}(y)$ where $q$ is the layer number along the $y$-axis, as shown in Fig. 2. Next, we assume the transverse bound modes are supported by the slab along the $y$ axis and propagate in the $x$-z plane. (e.g., suppose the propagation is along the z-axis). We further

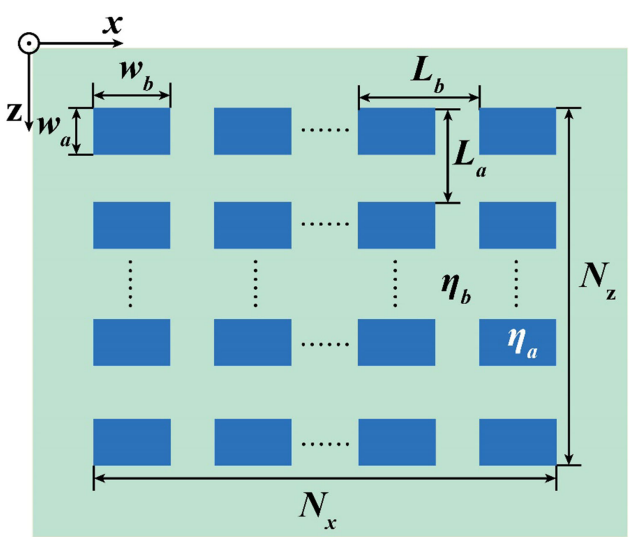

Fig. 3. Schematic of PC structure. $N_{x}$ and $N_{\mathrm{Z}}$ are number of periods along $x$ and $\mathrm{z}$ respectively.

assume the structure along the $y$ axis ensures a single bound mode $\beta=k_{0} \eta_{e f f, y}$, where $\beta$ is the propagation constant and $\eta_{\text {eff,y }}$ is the effective index of the mode The above assumptions are valid because in most laser structures, the active region has high refractive index and supports a bound mode to produce high photon density which is crucial in stimulated emission.

We note that any structural variations along the $y$ direction (e.g., a change in $\eta_{q}$ or thickness $d_{q}$ in any layer $q$ ) changes $\beta$ and hence changes $\eta_{e f f, y}$. In general, a change in $\eta_{q}$ or $d_{q}$ will produce a change in $\eta_{e f f, y}$. i.e., we assume that in general $\eta_{e f f, y}=\eta_{e f f, y}(x, z)$. Hence, by using this method, the $y$ direction structure is taken account of and replaced by an equivalent structure in $x$-z. i.e., $\eta_{e f f, y}=\eta_{e f f, y}(x, z)$.

A further assumption is that the mode along the $y$-axis is not significantly affected due to the physical structure $\eta(y ; x, z)$ and $d_{q}(x, z)$. The assumption is valid since in the PCSEL structure, the PC has a comparatively small index difference and acts as a perturbation. Thus, the problem is reduced to a 2-D $x$-z plane with $\eta_{e f f, y}=\eta_{e f f, y}(x, z)$ known, and the corresponding $x$-z plane geometrical structure given. For simplicity, we use $\eta_{e f f, y}(x, z)=\eta_{e f f}(x, z)=\left\{\eta_{a}, \eta_{b}\right\}$ in the following discussion.

\section{B. Modelling Technique: $x-z$ Plane}

Fig. 3. illustrate a schematic representation of the planar periodic structure in the $x$-z plane with a 2-D refractive index distribution $\eta(x, z)=\left\{\eta_{a}, \eta_{b}\right\}$. The periodicities are $L_{a}, L_{b}$ as shown and the number of periods along $x$ and $\mathrm{z}$ direction are $N_{x}, N_{z}$ respectively. The excitation is such that any non-zero field component, $\hat{F}(x, y, z)=\hat{F}(x, z)$ [23], i.e., $\partial / \partial y \approx 0$ is applicable. Then two types of field solution one with polarization $T E_{y} \Rightarrow E_{y}=0$ and the other with $T M_{y} \Rightarrow H_{y}=0$ can be sustained.

To be specific, for a typical inter-bond PCSEL, the $T E_{y}$ polarisation is important and is considered in the calculation since the typical active-layer gain medium (quantum well or quantum dots) supports this polarisation (electric dipole direction). From Maxwell's equation and by taking the approximation 


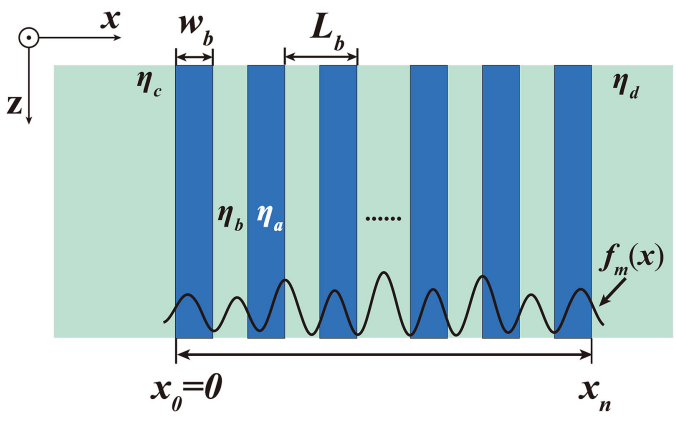

Fig. 4. Model description: lateral modes are computed for a relevant range of wavelengths.

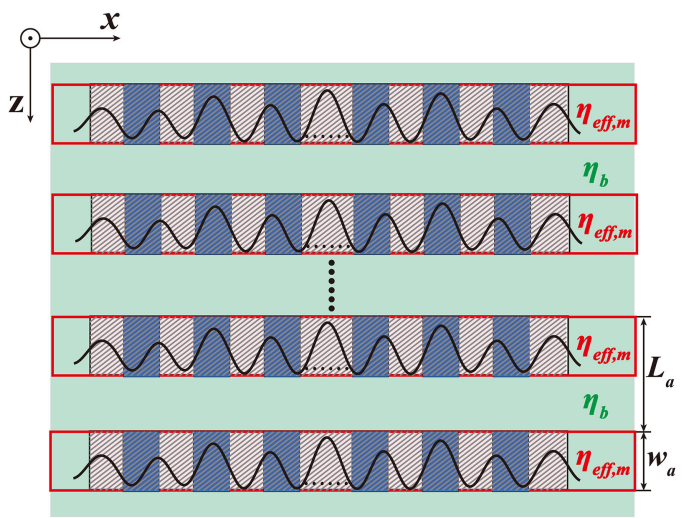

Fig. 5. The shaded area with dark blue region representing the multilayer waveguide in Fig. 3 is replaced by a homogeneous medium of effective modal index, resulting in a 1-D effective periodic grating along $\mathrm{z}$.

of $\mathbf{E} \cdot \nabla \varepsilon \sim 0$, any nonzero field component $\hat{F}_{m, n}(x, z)$ for the resonance modes ( $m, n$; integers) satisfied the acceptably approximate wave equation:

$$
\left[\frac{\partial^{2}}{\partial x^{2}}+\frac{\partial^{2}}{\partial z^{2}}+k_{0}^{2} \eta^{2}(x, z)\right] \hat{F}(x, z)=0
$$

Instead of expanding $\eta(x, z)$ and $\hat{F}(x, z)$ as Fourier series which is used in conventional techniques such as PWE and CMT, the procedure of obtaining a 2-D resonance $\hat{F}_{m, n}(x, z)$ begins by first considering index uniformity along the $\mathrm{z}$-direction. Then the structure becomes a periodic piecewise-constant multilayer structure along the z-direction as shown in Fig. 4. The eigenmode of such structure, $f_{m}(x)$ with the corresponding mode indices $\eta_{\text {eff, } m}$ are given by the wave equation:

$$
\left[\frac{\partial^{2}}{\partial x^{2}}+\frac{\partial^{2}}{\partial z^{2}}+k_{0}^{2} \eta^{2}(x)\right] F(x, z)=0
$$

where $F(x, z)=\sum_{m} f_{m}(x) e^{-j \beta_{m} z}$ and $\beta_{m}=k_{0} \eta_{e f f, m}$ represents the z-directed propagation constant. Since $\eta(x)$ satisfy the piece-wise constant condition, the eigenmode of such structure is evaluated over a pertinent range of wavelengths using the transfer matrix method [24], [25]. A detailed explanation of eigenmode evaluation of such structure is given in appendix.

Then, referring to Fig. 5, discontinuities along the z-axis are introduced to 'create' the 2-D PC structure corresponding

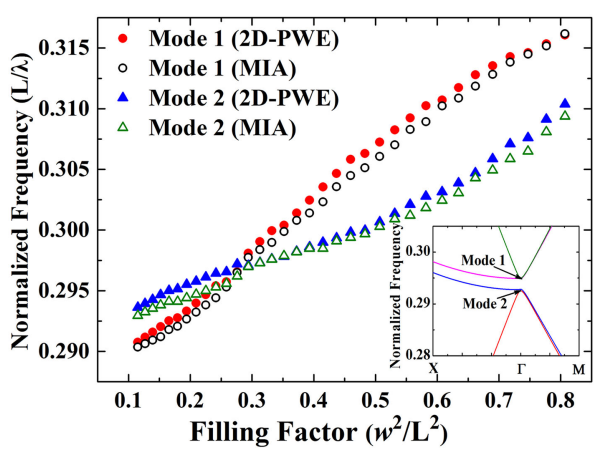

(a)

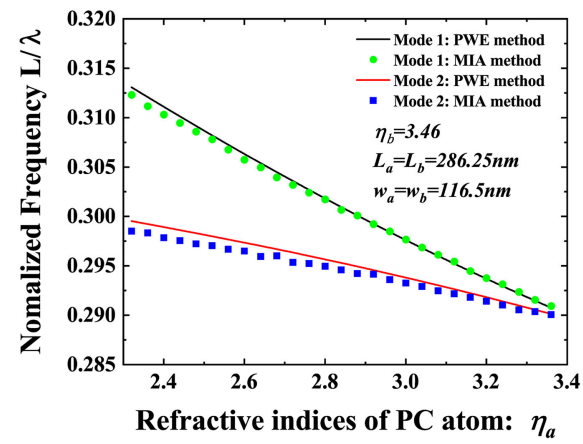

(b)

Fig. 6. (a) Band edge resonances calculated with varying filling factor. The insert figure is the photonic band structure near $\Gamma$ point calculated using PWE. (b) Band edge resonances calculated with varying $\eta_{a}$.

to Fig. 3 and thus the final resonance is then analysed as a waveguide discontinuity problem. In general, waveguide discontinuities excite all the modes of the structure including bound modes and radiation modes [26]. But in the present context of dimensions and magnitude of discontinuities, multimode generation at discontinuities [27] is approximated by self-mode conditions so that a simple MIA at discontinuities is used. Thus, with reference to Fig. 4, the field distribution $g_{n, m}\left[z ; f_{m}(x)\right]$ along the z-direction at resonance is obtained and the final result for the 2-D field distributions at resonances wavelength $\lambda_{0 \mathrm{~m}, n}$, are $\hat{F}(x, z)=f_{m}(x) g_{n, m}\left[z ; f_{m}(x)\right]$. The flow chart of the MIA is also given in the appendix.

\section{Validation}

To obtain the 2-D resonance, the resonance wavelength is searched over an appropriate range and the resultant resonances $\hat{F}_{m, n}(x, z)$ are obtained accordingly. Fig. 6(a) shows the band edge frequency as a function of filling factor. The solid red triangle and red circle are the band edge resonances calculated using PWE and the hollow triangle and circle are the first and second lasing modes calculated using MIA. The inset figure is the photonic band structure calculated using PWE. Note that only two non-leaky modes are compared with PWE since it is more favourable for lasing in PCSEL i.e., mode 1 or mode 2 of the photonic band structure in Fig. 6(a) [28].

When $\mathrm{N} \sim 10^{6}$ is used, (i.e., representing $N \rightarrow \infty$ ), band edge resonance calculated using MIA is compare with those 
I. Simulation Results
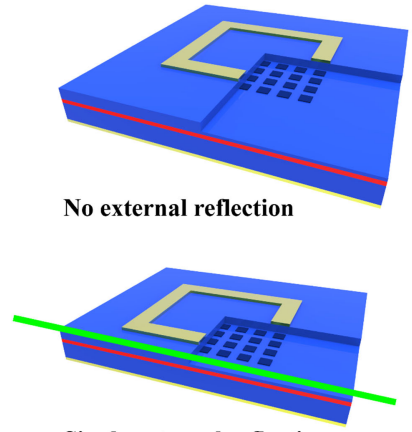

Single external reflection

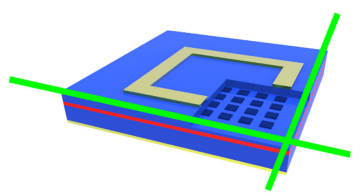

Double external reflection
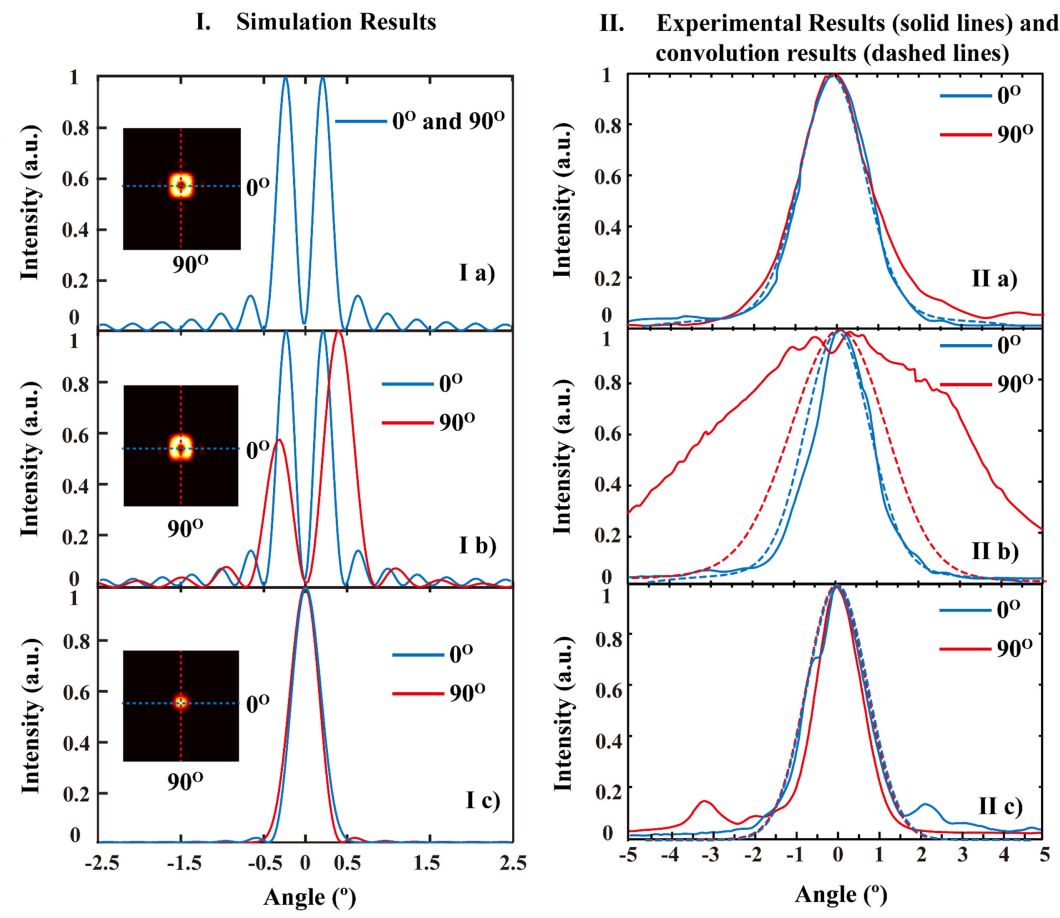

Fig. 7. far-field pattern changes accordingly as different external reflection are introduced: I a), I b), and I c) are simulated results; II a), II b), and II c) are modelling results after convolution with experimental results (dashed lines) and experimental results (solid lines).

calculated using PWE. As shown in Fig. 6(a), band edge modes obtained by this method match closely with those calculated using PWE over a large range of fill factor. Another comparison, by varying the index difference further allows a confirmation that MIA can correctly yield band edge resonances. Fig. 6(b) shows the band edge resonance as a function of refractive index of the background material. The black and red lines are the band edge resonance calculated using PWE and the triangles and squares are such values calculated using MIA. The essentially identical results further confirm that MIA matches well with conventional technique and is valid over a range of indices which are adequate to describe our all-semiconductor PCSEL devices.

It has therefore been shown in this section that the MIA method is versatile and yields very reliable results. This method is based on viewing the 2-D photonic crystal as a multilayer waveguide with periodic longitudinal discontinuities. Full use of the transfer matrix method makes the computation process computationally lean and fast. In the following, the MIA method is used to explain experimental results observed previously [12].

\section{EFFECT OF IN-PlanE FEEDBACK}

Experimental data of the PCSEL with external in-plane feedback has been reported previously [12], this feedback is introduced by cleaving at the edge of the photonic crystal region. In the experiment, one device was measured as fabricated, with a single cleave at one edge, and with two perpendicular cleaves at the edge (as shown as schematics in Fig. 7). The operation is performed to ensure the cleave is along the edge of photonic crystal area and the number of periods is not affected during the process. It was shown that when a single cleave is introduced to the sample, the threshold of the PCSEL was reduced and the introduction of the second cleave did not reduce the threshold current further. In both of the single and double cleave experiments, single mode operation was maintained but the cleave lead to significant changes of the farfield pattern, as shown in Fig. 7.

We note that the cleave of the PCSEL effectively truncates the periodic structure with a different boundary (from semiconductor to air rather than from semiconductor to semiconductor). This effect can be readily investigated approximately using MIA. Note that although in [12], the fabricated device has a triangular-shaped photonic crystal, the approximation is such that the 2-D resonance is mainly given by the overall periodicity and the shape of photonic crystal will have more effect on local field distribution rather than resonance wavelength. As shown in Fig. 7, MIA is used for the simulation of a PCSEL device subject to in-plane feedback (green lines) from cleaved facets at the PC boundary. Column I shows far-field patterns simulated using the MIA technique for the PCSEL (with PC $500 \times 500$ period) used in our experimental study. Column III shows experimental data reported previously and the simulated structure is described previously [12].

By comparison to experiment, a narrower farfield pattern is observed in simulation. Note that such analysis is very approximate since rather than a rigorous diffraction analysis, we treat the problem as an emission problem. The vertical dimension is not considered at the first stage, instead, source field $\hat{F}(x, z)$ which is readily obtained using MIA is used and an emission analysis is carried out to obtain far-field distribution. Column II with dashed lines shows the data from column I convoluted with a Gaussian function (FWHM of each peak in column I is broadened by a factor of 2, mimicking the effect of fabrication and experimental 


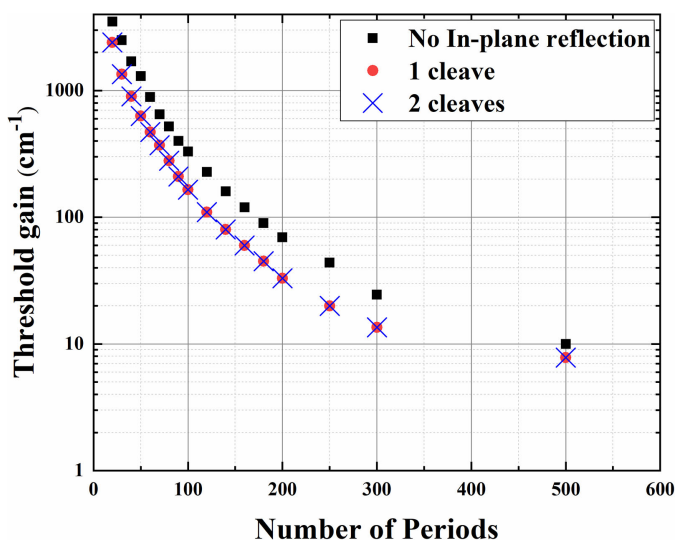

Fig. 8. MIA modelling result of threshold gain of PCSEL with and without single or double external reflection.

imperfection). The simulation reproduces much of the character of the experimental data (presented in Column II, solid lines), in particular reproducing the increase in far-field angle and asymmetry of the beam for one feedback facet.

Fig. 8 shows the change of threshold gain as a function of $\mathrm{PC}$ period, for the different external feedback conditions discussed in the previous section (black square: no-feedback, red circle: one-feedback, black cross: double feedback). It is shown that a single cleave along the edge of PC region results in a reduction of threshold while the second cleave does not change the threshold significantly. Thus, MIA has the potential to explain the phenomenon in our previous experimental results [12]. Note that mode competition as observed in previous experiment is not seen in the model. This is because in MIA, higher-order effects are neglected, and the carrier effect are not included.

Fig. 9 shows the near field distribution of the lasing mode subject to in-plane feedback for the case of no feedback, one, and two cleaves, The simulation shows that the peak in the near-field pattern is displaced toward the cleaved facet(s). This displacement amounts to several 10's of $\mu \mathrm{m}$ for the case simulated here (reflectivity given by semiconductor/air interface, $500 \times$ 500 periods). These results indicate that the mode position and shape can be controlled through the in-plane external feedback, opening the route to all-electronic beam steering and beam shaping.

\section{PCSEL AREA ScAling AND In-Plane FEEDBACK}

The design of a semiconductor laser depends on its specific application. Broadly speaking, high power, narrow beam and monochromatic light are three highly desirable characteristics of semiconductor laser. It is known that large active area VCSEL devices lead to multi-transverse mode emission. Due to the 2-D feedback in transverse directions, PCSELs can support largescale single-mode emission.

However, the transverse dimension needs to be large enough to provide sufficient feedback for a reasonable threshold (300500 periods in a typical PCSEL device [5], [28]). The large lateral dimension of the PCSEL enables power scaling, and diffraction limited divergence, but limits use in applications that

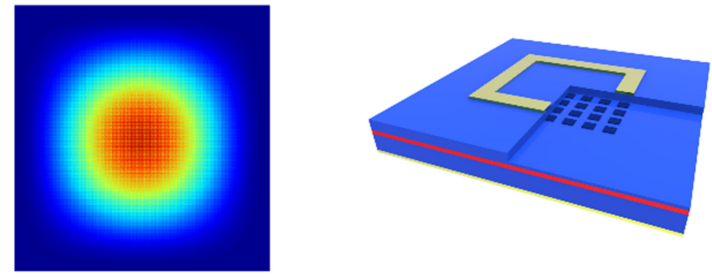

(a) No cleave

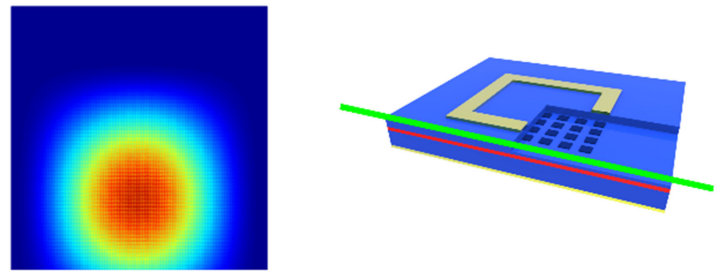

(b) Single cleave

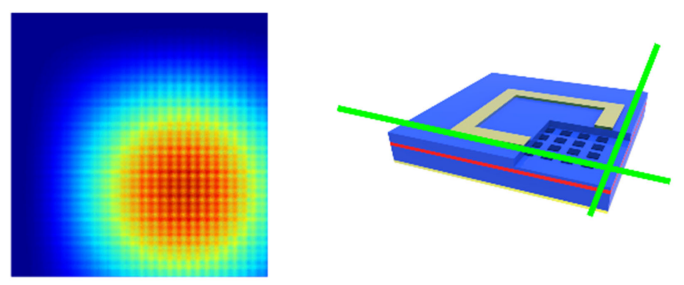

(c) Double cleave

Fig. 9. In-plane field distribution of lasing mode of PCSEL with and without single or double external reflection.

require high speed modulation due to the high mode volume and parasitic capacitance of the junction. Thus, a simulation-based study to explore the opportunities to reduce the scale of the PCSEL, incorporating different boundary conditions is timely. It has been discussed in the previous section that compared with conventional technique such as PWE, finite size effects can be readily obtained from the MIA method.

The threshold margin is defined as the difference in gain between the resonance mode with lowest threshold gain and the mode with next lowest threshold gain [29]. The gain margin is important because it represents how stable the lasing will be under high speed modulation. Fig. 10 plots the gain margin as a function of PC period for $R=|r|^{2}=70 \%$ with phase $\phi=7 \pi / 8 . r$ is the field reflectivity $r=|r| \exp (i \phi)$. This preliminary modelling result shows that the threshold gain margin can be increased through the reduction of the photonic crystal area, whilst incorporating in-plane external feedback with this reflectivity and phase. For device operation, it is ideal to have large threshold margin for stable lasing operation, especially under high speed modulation [30]. We note that the reduction of PCSEL area results in an increase of the gain margin.

Fig. 11 shows the lasing threshold of the modelled PCSEL as a function of number of photonic crystal period. The external reflection is place along four edges of the $\mathrm{PC}$ region with the field reflectance varying from $30 \%$ to $90 \%$. It is noticed that a reduction of PC period results in an increasing of the lasing threshold due to the smaller in-plane feedback and the leakage 


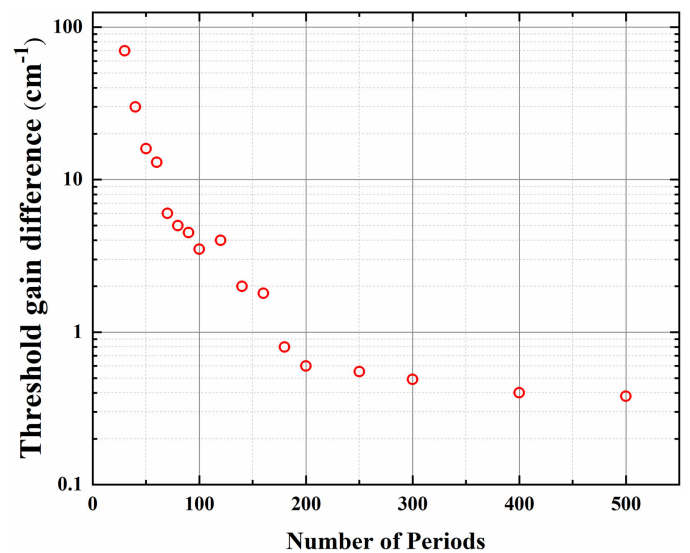

Fig. 10. Threshold gain margin with different number of period.

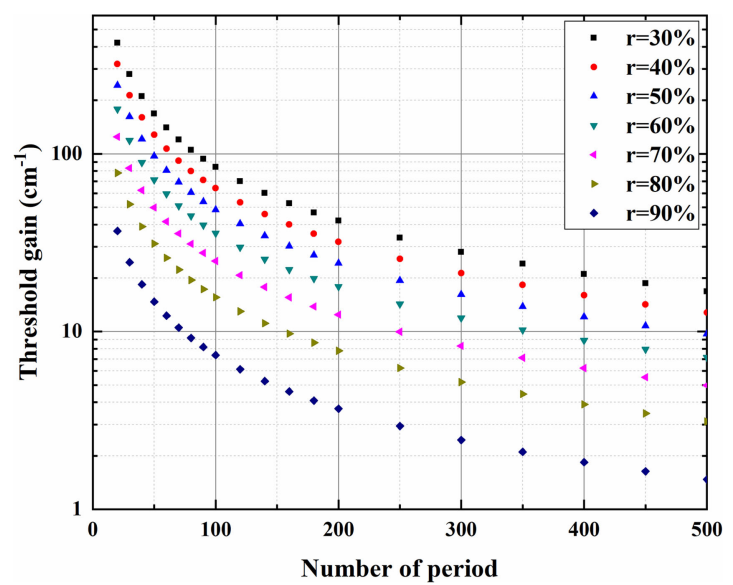

Fig. 11. Threshold gain margin with different number of period and different external reflectance strength. The reflectance value represents field reflection and the feedback is at four sides of PC region.

of power to the unpumped non-PC regions. It is also noticed that such a drawback can be removed through adding external in-plane feedback to increase the $\mathrm{Q}$ factor. In practice, the external feedback can be added through specifically designed high reflectors such as a Bragg grating, or first order PCs.

Fig. 12 shows the phase effect of the external reflection at $70 \%$ of field reflectivity, the threshold gain of PCSEL is plotted as a function of reflection phase variation with different period (red circle, $N=200$, black square, $N=180$ ). For the band edge resonance (lasing mode), the threshold gain varies as the phase shift is varied from 0 to $2 \pi$, the threshold gain reaches a minimum at $7 \pi / 8$ and a maximum at $3 \pi / 2$. As may be expected, reflection phase of in-plane feedback plays an important role in determining the increasing or decreasing of lasing threshold. It also suggests that threshold gain can be optimized with suitable facet phase reflectivity.

Fig. 13 illustrates for $70 \%$ of reflectivity, the band edge resonance modes of PCSEL, plotted as a function of reflection phase. It is shown from the model that although lasing still occurs at two band-edge, the lasing mode change while varying the reflection phase. Such phenomenon has also been observed and studied

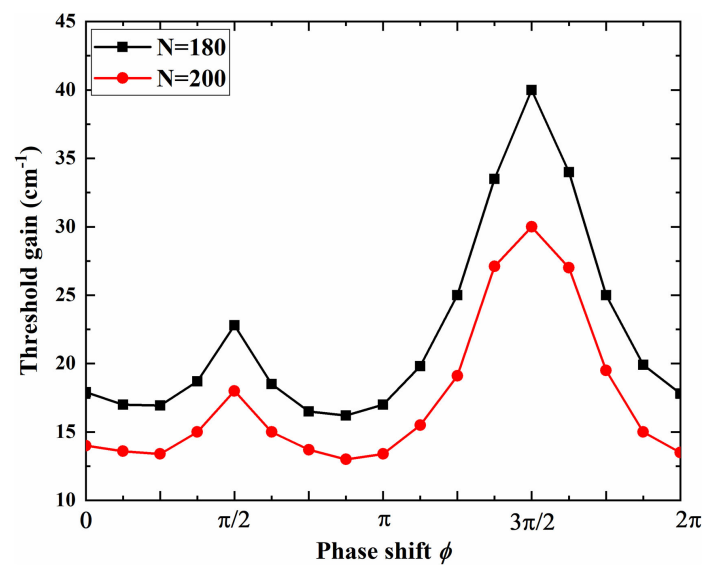

Fig. 12. Threshold gain with different number of period and different external reflectance phase.

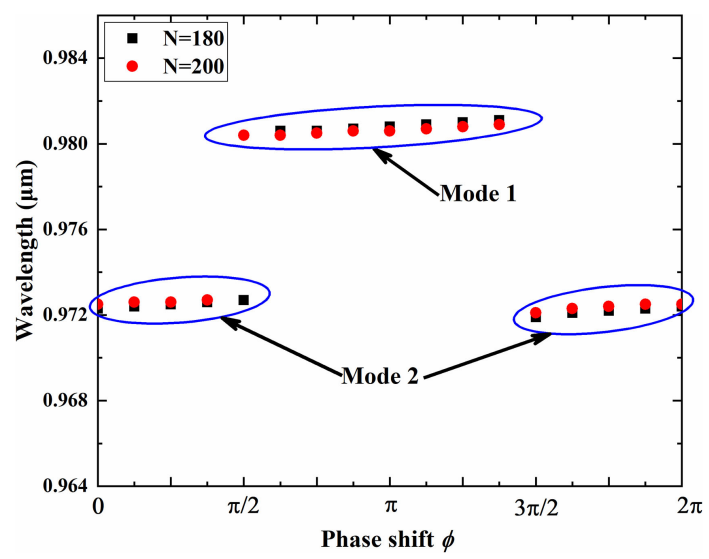

Fig. 13. Lasing wavelength for PCSEL with varying phase of external reflection.

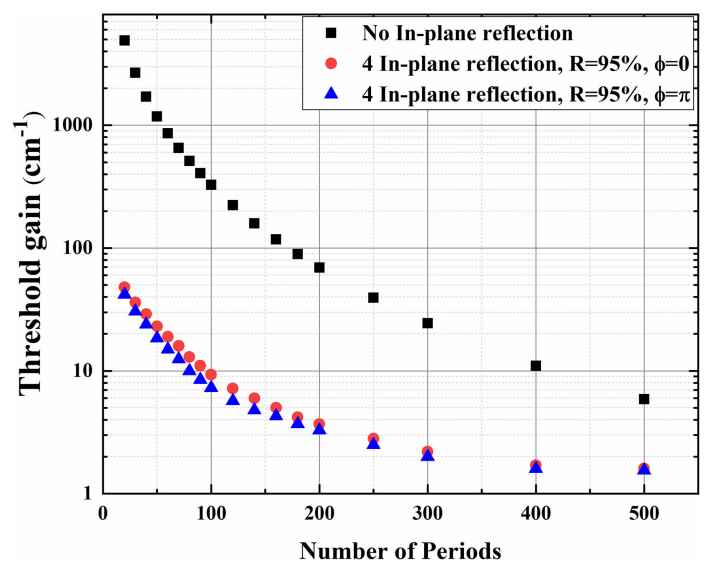

Fig. 14. Threshold gain of PCSEL with different number of period and different external in-plane phase reflectivity.

in conventional DFB lasers [17] and this effect can be further investigated to control the lasing mode of a PCSEL through external in-plane feedback. However, a more comprehensive modelling based on MIA include, for example, spatial and temporal gain distribution and other PC configurations are also required to study mode selection mechanism in PCSEL. 
The study of PCSEL threshold gain with external feedback indicates the possibility of device geometry with small PC active region. As shown in Fig. 14, an example of the threshold gain of a PCSEL is plotted as a function of number of period at 0 (black square) and $90 \%$ reflection with reflection phase at 0 (red circle) and $\pi$ (blue triangle). It is shown that with appropriate reflection strength and phase, the PCSEL may be shrank down to $20 \times 20$ period with a reasonable threshold gain of $50 \mathrm{~cm}^{-1}$.

\section{CONCLUSION}

In this work, a novel, modal index analysis method has been used for analysing experimental results of PCSEL devices presented previously. The computation process is based on wave propagating in periodic multilayer media which is quasi-analytic and hence computationally lean. The comparison with numerical and experimental results shows that this method yields very reliable results. The MIA technique has been used to study the PCSEL with different periodicity and boundary reflection. It is shown that by carefully designing external reflector, the dimension of the PCSEL can be shrink down and the lasing characteristic such as threshold, frequency and mode shape can be controlled accordingly.

\section{APPENDIX}

As shown in Fig. 4 , in the multilayer regions, $\eta_{a}, \eta_{b}$ such that $\kappa_{a}^{2}+\beta^{2}=k_{0}^{2} \eta_{a}^{2}$ and $\kappa_{b}^{2}+\beta^{2}=k_{0}^{2} \eta_{b}^{2}$ where $\kappa$ and $\beta$ represent the $x$-directed and $\mathrm{z}$-directed propagation constants in the corresponding regions. Applying the usual field matching conditions at the abrupt interfaces [16] between regions ' $a$ ' and 'b' yields the elements of the $2 \times 2$ unimodular transfer matrix, $\mathbf{m}$, for a typical unit cell [16].

Further, and very importantly, the periodic nature of the multilayer medium is utilised to obtain an efficient formulation for the composite transfer matrix accounting for the corresponding number, $N$, of periodic layers [30]. Thus, the total transfer matrix is given by $\mathbf{M}_{T}=\mathbf{m}^{N}$ where $\mathbf{m}$ is the unimodular transfer matrix:

$$
\begin{array}{r}
\mathbf{M}_{T}=\mathbf{m}^{N}=\left(\begin{array}{cc}
m_{11} m_{12} \\
m_{21} m_{22}
\end{array}\right)^{N} \\
=\left(\begin{array}{rc}
m_{11} U_{N-1}-U_{N-2} & m_{12} U_{N-1} \\
m_{21} U_{N-1} & m_{22} U_{N-1}-U_{N-2}
\end{array}\right)
\end{array}
$$

$m_{11}, m_{12}, m_{21}$ and $m_{22}$ are the matrix elements, and $U_{N}$ are known as Chebyshev polynomials of the second kind:

$$
U_{N}=\frac{\sin \left[(N+1) \cos ^{-1}\left(\frac{m_{11}+m_{22}}{2}\right)\right]}{\sin \left[\cos ^{-1}\left(\frac{m_{11}+m_{22}}{2}\right)\right]}
$$

There are many different methods to calculate power $\mathrm{N}$ of unimodular $2 \times 2$ matrix [24], [30] which enables analysing a truncated periodic structure since, unlike infinite periodic structures, the Bloch theorem does not strictly apply in this situation [24]. This procedure is also beneficial since it provides for a significantly reduced computation time. The resonant mode for the entire multilayer (periodic) structure can be calculated from the total transfer matrix, which effectively is the solution of a transcendental equation: $\left|m_{11} U_{N-1}-U_{N-2}\right|=1$. Any

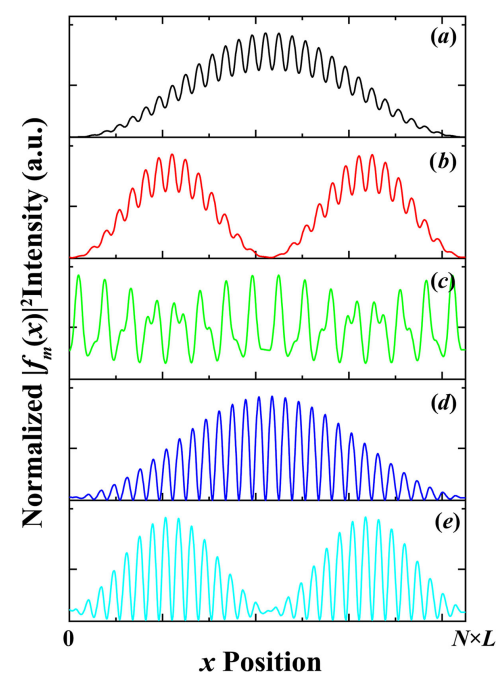

Fig. 15. Examples of eigen-mode solutions $f_{m}(x)$ of 1-D periodic structure (Fig. 4).

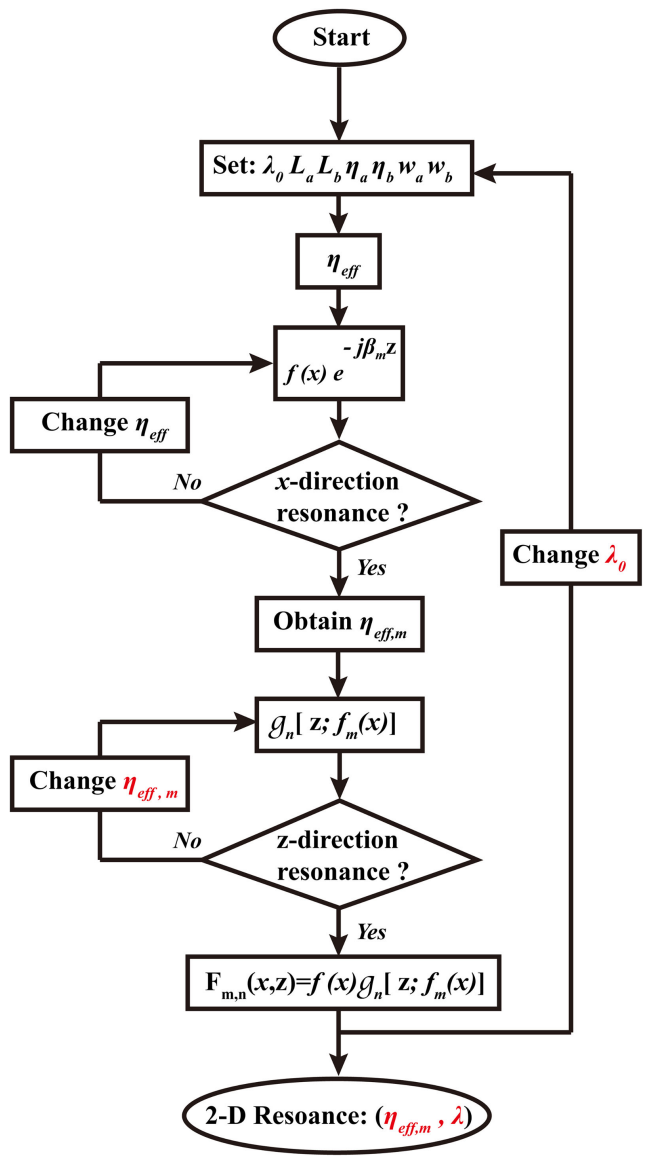

Fig. 16. Flowchart of the modelling process.

change in the boundary can be introduced as a change in matrix elements.

For a relevant range of wavelengths, eigenmode of such 1-D periodic structure is calculated. An example of the corresponding typical field distributions (labelled $a, b, c, d$ and $e$ ) of such 1-D periodic structure are shown in Fig. 15, with $N_{x}=\mathrm{N}=30$. $L_{b}=290 \mathrm{~nm}, w_{b}=115 \mathrm{~nm}, \eta_{a}=3.1, \eta_{b}=3.5$. 
Optical gain is introduced as an imaginary part of refractive index which can be included in the matrix formulation. It is also assumed that there is no spatial or temporal distribution of gain. Note that typical imaginary part of refractive $\left(\sim 10^{-2}\right)$ is very small compared to the real part, the mode pattern is not affected when varying the optical gain.

For threshold gain, after calculate $\eta_{e f f, m}=\eta_{r}+j \eta_{i}$, which is complex in general, the structure become 1-D periodic structure in z-direction (Fig. 5) with 2-D effect. The lasing threshold of such effective structure is taken by solving $\left|\mathbf{M}_{T(1,1)}\right|=0$ where $\mathbf{M}_{T(1,1)}$ is the matrix element of the new transfer matrix $\mathbf{M}_{T}$ of the new effective structure. Note that this is also the oscillation condition (lasing threshold) in 1-D DFB laser [29]. Indeed, the solution of this equation is complex. So both real and imaginary part of the solution should equal to zero. At such condition, the corresponding imaginary part of refractive index is regarded as our lasing threshold $g_{t h}=k_{0} \eta_{i}$ where $k_{0}$ is the wavevector in vacuum.

\section{ACKNOWLEDGMENT}

The author would like to thank Dr. Timothy S. Roberts, Dr, Benjamin J. Stevens and Mr. Bret Harrison in University of Sheffield for semiconductor regrowth. We would like to thank Dr. Nasser Babazadeh for device fabrication and preparation, and thank Dr. Gary Terrnent for e-beam lithography.

\section{REFERENCES}

[1] J. Coleman, "The development of the semiconductor laser diode after the first demonstration in 1962," Semicond. Sci. Technol., vol. 27, no. 9, 2012, Art. no. 090207.

[2] E. Kapon, Semiconductor Lasers I: Fundamentals. London, U.K.: Academic Press, 1999.

[3] K. Hirose et al., "Watt-class high-power, high-beam-quality photoniccrystal lasers," Nature Photon., vol. 8, no. 5, p. 406, 2014.

[4] E. Miyai et al., "Photonics: Lasers producing tailored beams," Nature, vol. 441, no. 7096, p. 946, 2006.

[5] D. M. Williams et al., "Epitaxially regrown gaas based photonic crystal surface emitting laser," in Proc. IEEE Photon. Conf., 2011, pp. 705-706.

[6] M. Meier et al., "Laser action from two-dimensional distributed feedback in photonic crystals," Appl. Phys. Lett., vol. 74, no. 1, pp. 7-9, 1999.

[7] K. Iga, "Surface-emitting laser-its birth and generation of new optoelectronics field," IEEE J. Sel. Topics Quantum Electron., vol. 6, no. 6, pp. 1201-1215, Nov./Dec. 2000.

[8] D. Ohnishi, T. Okano, M. Imada, and S. Noda, "Room temperature continuous wave operation of a surface-emitting two-dimensional photonic crystal diode laser," Opt. Express, vol. 12, no. 8, pp. 1562-1568, 2004.

[9] S. Noda, M. Yokoyama, M. Imada, A. Chutinan, and M. Mochizuki, "Polarization mode control of two-dimensional photonic crystal laser by unit cell structure design," Science, vol. 293, no. 5532, pp. 1123-1125, 2001.

[10] R. Taylor et al., "Electronic control of coherence in a two-dimensional array of photonic crystal surface emitting lasers," Scientific Rep., vol. 5 , 2015, Art. no. 13203.

[11] R. J. E. Taylor et al., "Coherently coupled photonic-crystal surfaceemitting laser array," IEEE J. Sel. Topics Quantum Electron., vol. 21, no. 6, pp. 493-499, Nov./Dec. 2015.

[12] R. J. Taylor et al., "Mode control in photonic crystal surface emitting lasers through external reflection," IEEE J. Sel. Topics Quantum Electron. vol. 23 , no. 6 , pp. 1-8, Nov./Dec. 2017.

[13] J. D. Joannopoulos, S. G. Johnson, J. N. Winn, and R. D. Meade, Photonic Crystals: Molding the Flow of Light, 2nd ed. Princeton, NJ, USA Princeton Univ. Press, 2011.

[14] L. Brillouin, Wave Propagation in Periodic Structures: Electric Filters and Crystal Lattices. New York, NY, USA: Dover, 1953.
[15] L. Rayleigh, "XVII. On the maintenance of vibrations by forces of double frequency, and on the propagation of waves through a medium endowed with a periodic structure," The London, Edinburgh, Dublin Philos. Mag. J. Sci., vol. 24, no. 147, pp. 145-159, 1887.

[16] L. A. Coldren, S. W. Corzine, and M. L. Mashanovitch, Diode Lasers and Photonic Integrated Circuits. Hoboken, NJ, USA: Wiley, 2012.

[17] J. E. Carroll, J. Whiteaway, and D. Plumb, Distributed Feedback Semiconductor Lasers. London, U.K.: Institution of Elect. Engineers, 1998.

[18] M. Plihal and A. A. Maradudin, "Photonic band structure of twodimensional systems: The triangular lattice," Physical Rev. B, vol. 44, no. 16 , pp. 8565-8571, 1991

[19] I. Vurgaftman and J. R. Meyer, "Design optimization for high-brightness surface-emitting photonic-crystal distributed-feedback lasers," IEEE J. Quantum Electron., vol. 39, no. 6, pp. 689-700, Jun. 2003.

[20] S. Fan and J. D. Joannopoulos, "Analysis of guided resonances in photonic crystal slabs," Physical Rev. B, vol. 65, 2002, Art. no. 235112

[21] L. C. Botten, N. A. Nicorovici, R. C. McPhedran, C. M. Sterke, and A. A. Asatryan, "Photonic band structure calculations using scattering matrices," Phys. Rev. E Stat. Nonlinear Soft Matter Phys., vol. 64, no. 4 (Pt 2), Oct. 2001, Art. no. 046603

[22] G. Li, J. Sarma, and R. Hogg, "Evaluating resonances in PCSEL structures based on modal indices," IET Optoelectron., vol. 13, no. 1, pp. 17-22, Feb. 2019.

[23] H. Han and J. Coleman, "Two-dimensional rectangular lattice distributed feedback lasers: A coupled-mode analysis of TE guided modes," IEEE J. Quantum Electron., vol. 31, no. 11, pp. 1947-1954, Nov. 1995.

[24] P. Pereyra, "Theory of finite periodic systems: The eigenfunctions symmetries," Ann. Phys., vol. 378, pp. 264-279, 2017.

[25] A. Ghatak, K. Thyagarajan, and M. Shenoy, "Numerical analysis of planar optical waveguides using matrix approach," J. Lightw. Technol., vol. 5, no. 5, pp. 660-667, May 1987.

[26] T. E. Rozzi, "Rigorous analysis of the step discontinuity in a planar dielectric waveguide," IEEE Trans. Microw. Theory Techn., vol. 26, no. 10, pp. $738-746$, Oct. 1978.

[27] A. Ishimaru, Electromagnetic Wave Propagation, Radiation, and Scattering: From Fundamentals to Applications. Hoboken, NJ, USA: Wiley, 2017.

[28] K. Sakai et al., "Lasing band-edge identification for a surface-emitting photonic crystal laser," IEEE J. Sel. Areas Commun., vol. 23, no. 7, pp. 1335-1340, Jul. 2005.

[29] G. Bjork and O. Nilsson, "A new exact and efficient numerical matrix theory of complicated laser structures: Properties of asymmetric phaseshifted DFB lasers," J. Lightw. Technol., vol. 5, no. 1, pp. 140-146, Jan. 1987.

[30] A. Yariv and P. Yeh, Photonics: Optical Electronics in Modern Communications. London, U.K.: Oxford Univ. Press, 2007.

Guangrui Li received the B.Eng. degree in electrical engineering and automation from Fudan University, Shanghai, China, in 2013, and the M.Sc. degree in semiconductor photonics from the University of Sheffield, Sheffield, U.K., in 2014. From 2014 to 2016, he did the postgraduate course from the University of Sheffield. He is currently working toward the Ph.D. degree in photonic crystal surface emitting lasers with the University of Glasgow, Glasgow, U.K.

Jayanta Sarma received the B.Eng. degree in electronics and telecommunication engineering from Jadavpur University, Kolkata, India, the M.S. degree in electrical engineering from the Illinois Institute of Technology, Chicago, IL, USA, and the Ph.D. degree from the Department of Electronics \& Electrical Engineering, University of Leeds, Leeds, U.K. He was then a National Research Council of Canada Post-doctoral Research Fellow with the Communications Research Centre, Ottawa, ON, Canada. He then returned to the U.K. and worked as a Research Associate with the Universities of Sheffield, Liverpool, and Bath, U.K. He was appointed a Member of Academic Staff at the University of Bath, where he led a research group working on guided-wave photonics until he retired as Reader from the university. He is a Visiting Researcher with the Department of Electronic and Electrical Engineering, The University of Glasgow, Glasgow, U.K. 
Richard J. E. Taylor received the B.Eng. degree in electrical engineering, the M.Sc. degree in semiconductor photonics, and the Ph.D. degree in photonic crystal surface emitting lasers from the Department of Electronic and Electrical Engineering, The University of Sheffield, Sheffield, U.K., in 2010, 2011, and 2015 , respectively. Upon completion of the Ph.D. degree, he received the EPSRC Doctoral Prize Fellowship from the University of Sheffield. From 2015 to 2017, he was a JSPS Post-doctoral Research Fellow with the University of Tokyo, Tokyo, Japan. Since 2017, he has been a Post-doctoral Research Associate with the Electronic and Nanoscale Engineering Group, School of Engineering, University of Glasgow, Glasgow, U.K.

David T. D. Childs (M'15) received the B.Sc. degree in physics, the M.Sc. degree in semiconductor science and technology, and the Ph.D. degree in properties and applications of $1.3 \mu \mathrm{m} \mathrm{InAs/GaAs} \mathrm{quantum} \mathrm{dot} \mathrm{devices} \mathrm{from} \mathrm{Imperial} \mathrm{College,}$ London, U.K., in 1996, 1997, and 2002, respectively.

He was then with the R\&D Department of Marconi Optical Components (later Bookham, now Oclaro), Caswell Semiconductor Research and Fabrication, until 2006, where he was responsible for the development of a range of telecoms lasers. During this time, he also worked on several European projects developing quantum dot technology. Following this, he joined the Department of Electronic and Electrical Engineering, University of Sheffield, Sheffield, U.K., where he was engaged in a number of projects developing semiconductor light sources from visible through to $\mathrm{THz}$ wavelengths. He was also involved in developing systems to demonstrate the application of semiconductor devices to fields ranging from selective laser melting (3-D printing) to mid-infrared hyperspectral imaging (biomedical imaging). Since 2015, he has been a Lecturer with the Electronic and Nanoscale Engineering Group, School of Engineering, The University of Glasgow, Glasgow, U.K. He has contributed to over 100 journal and conference publications. His research interests span from semiconductor light emitter development through photonic integration to the applications of these devices and systems from communications to biomedicine.
Richard A. Hogg received the Ph.D. degree in physics from the University of Sheffield, Sheffield, U.K., in 1995, and then spent two years as a Post-doctoral Researcher with the NTT Basic Research Laboratories, Atsugi, Japan.

He was then awarded an EU-Japan Fellowship as a Visiting Researcher with Professor Arakawa's Laboratory, University of Tokyo. He subsequently spent three years with Toshiba Research Europe's Cambridge Laboratory before moving to Agilent Technologies Fibre-Optic Component Operation, Ipswich, U.K., in 2000. In 2003, he joined the Electronic and Electrical Engineering Department, University of Sheffield. In 2015, he became a Professor of electronic and nanoscale engineering with the University of Glasgow, Glasgow, U.K. His research group is active in developing the understanding of device physics and engineering, epitaxial processes, fabrication technologies, and applications of various semiconductor laser, amplifier, and superluminescent diode devices. 